\title{
Rational Band Engineering of Organic Double-Heterojunction for Artificial Synaptic Device with Enhanced State Retention and Linear Update of Synaptic Weight
}

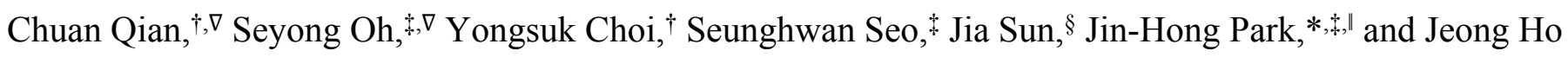
Cho*,†

†Department of Chemical and Biomolecular Engineering, Yonsei University, Seoul 120-749, Republic of Korea.

Department of Electrical and Computer Engineering, Sungkyunkwan University, Suwon 16419, Republic of Korea.

$\S$ Hunan Key Laboratory for Super Microstructure and Ultrafast Process, School of Physics and Electronics, Central South University, Changsha, Hunan 410083, P. R. China.

"SKKU Advanced Institute of Nanotechnology (SAINT), Sungkyunkwan University, Suwon 16419, Republic of Korea.

\section{Corresponding Authors}

*E-mail: jhcho94@yonsei.ac.kr.

*E-mail: jhpark9@skku.edu. 


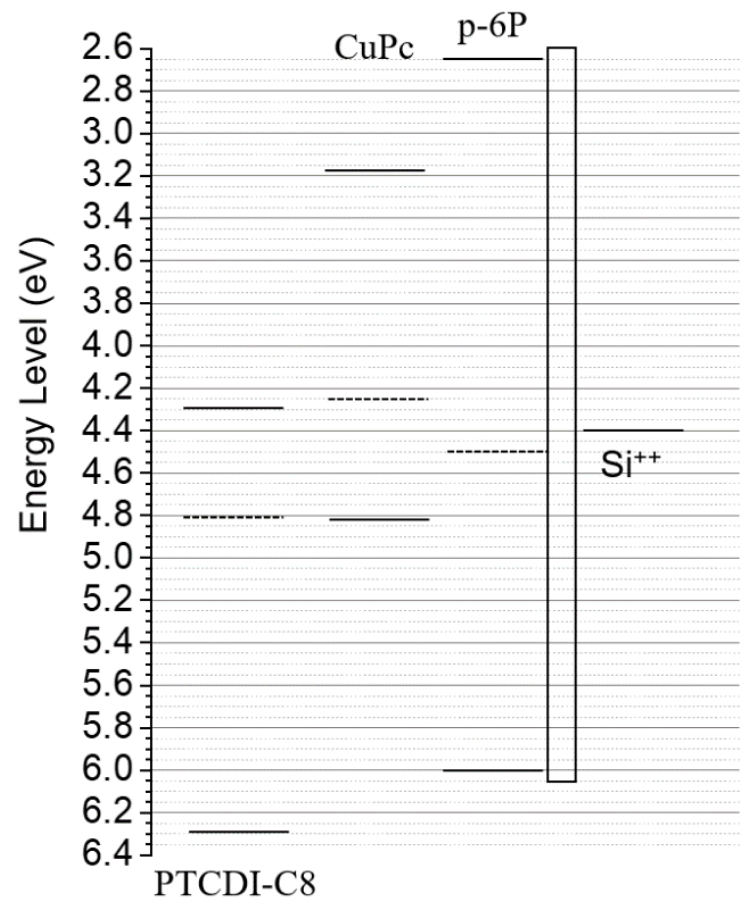

Figure S1. Numerical information for aligning the energy bands of PTCDI- $\mathrm{C}_{8}, \mathrm{CuPc}, p-6 \mathrm{P}, \mathrm{SiO}_{2}$, and $\mathrm{Si}^{++}$.
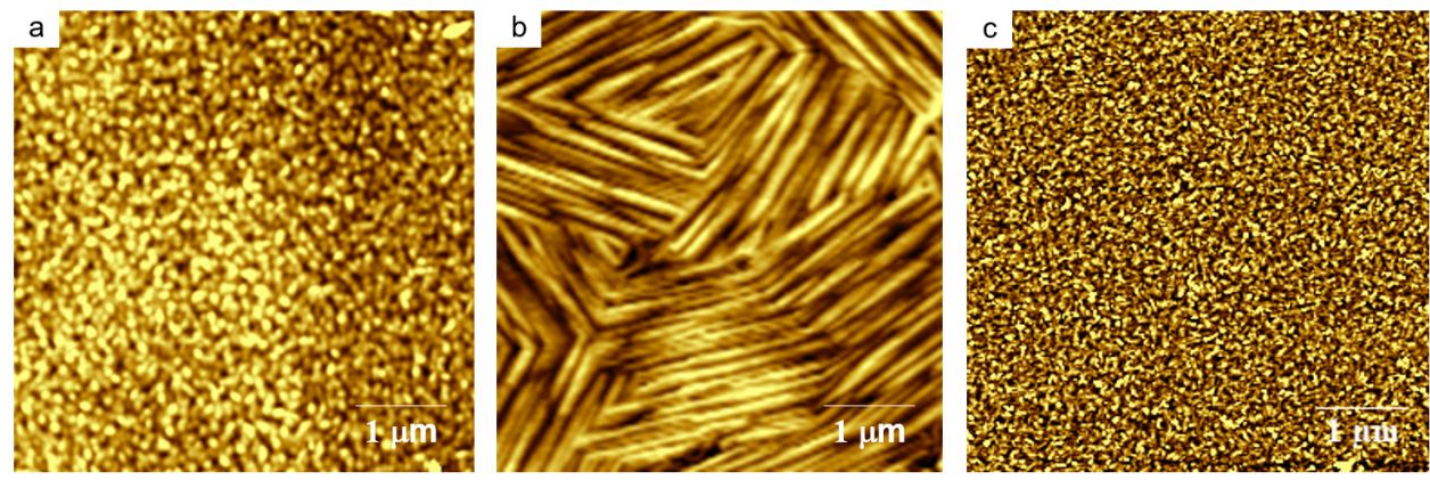

Figure S2. AFM images for (a) $\mathrm{CuPc} / \mathrm{SiO}_{2}$, (b) $\mathrm{CuPc} / p-6 \mathrm{P}$ and (c) $\mathrm{PTCDI}-\mathrm{C}_{8} / \mathrm{CuPc}$ film. 


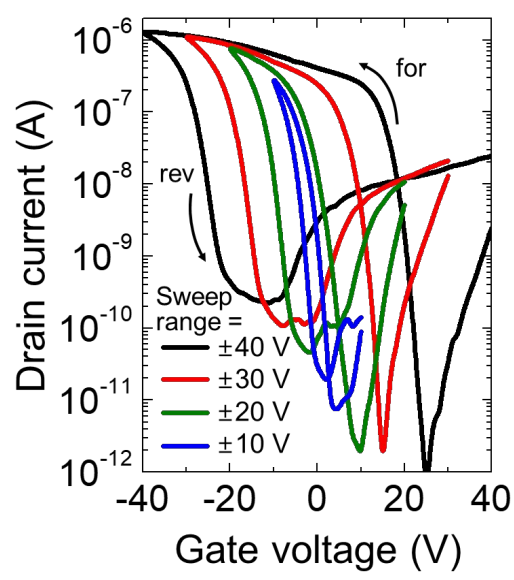

Figure S3. Transfer characteristics of the PTCDI-C $8 / \mathrm{CuPc} / p-6 \mathrm{P}$ synaptic device under various $V_{\mathrm{G}}$ sweep ranges.

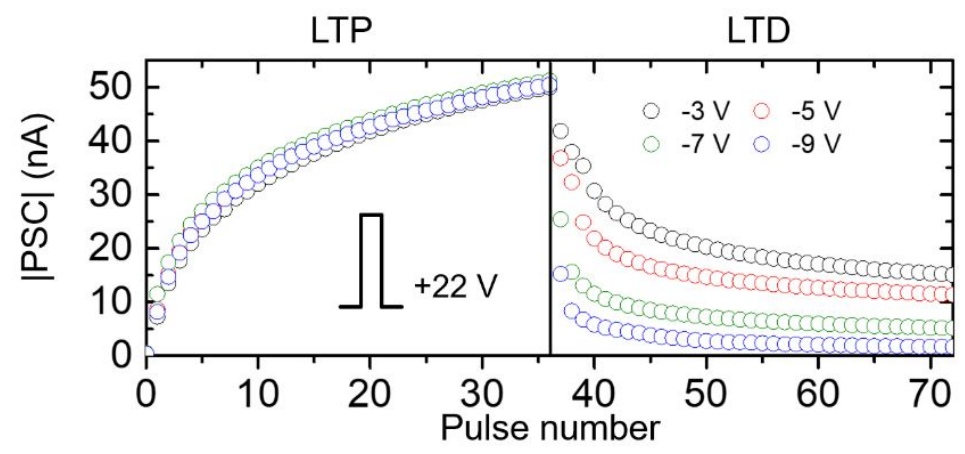

Figure S4. LTP/LTD characteristics of the PTCDI- $\mathrm{C}_{8} / \mathrm{CuPc} / p-6 \mathrm{P}$ synaptic device with 36 pulses of $+22 \mathrm{~V}$ and 36 pulses of negative $V_{\mathrm{WC}} \mathrm{S}(-3,-5,-7$, and $-9 \mathrm{~V})$.
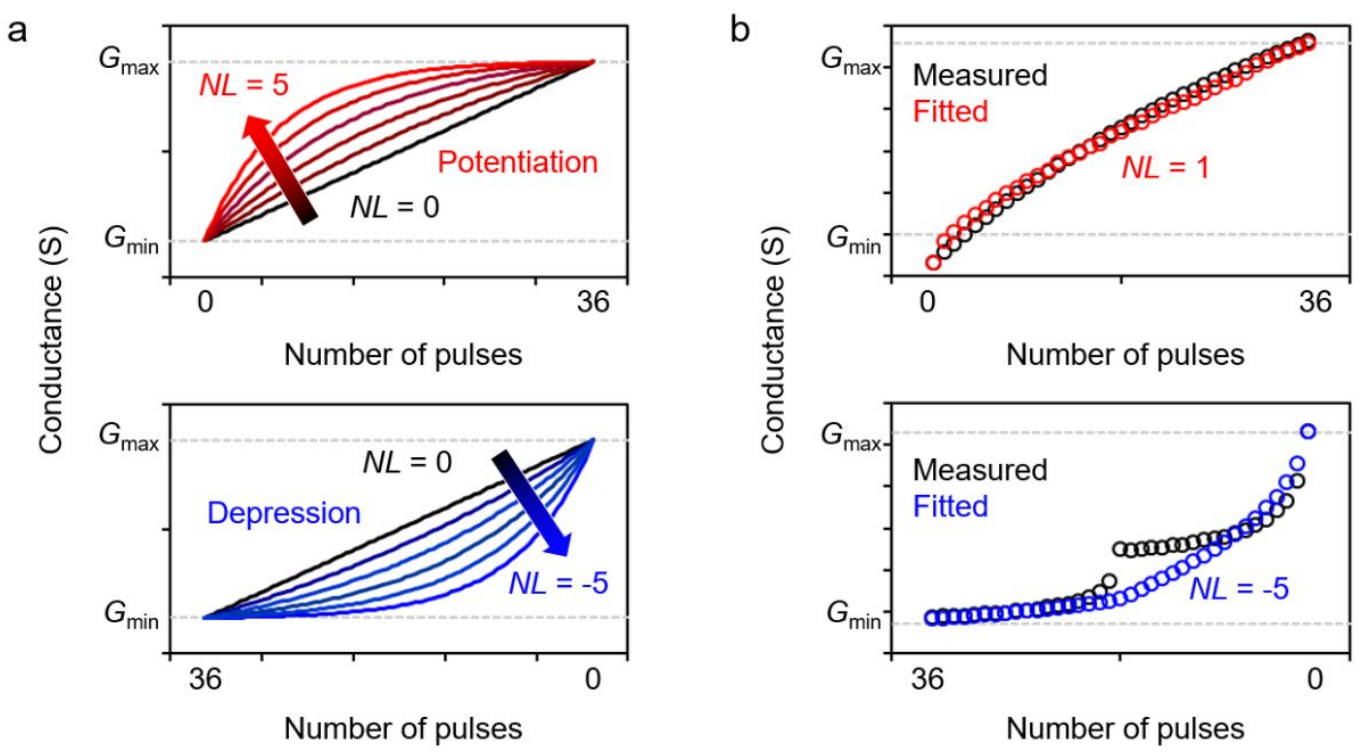

Figure S5. Nonlinearity analysis of the LTP/LTD characteristic curves. (a) LTP and LTD with respect to the nonlinearity (NL), ranging from 0 to 5. (b) Fitting curves of both LTP and LTD using the extracted parameters given at $\mathrm{NL}_{\mathrm{P} / \mathrm{D}}=1 /-5$. 


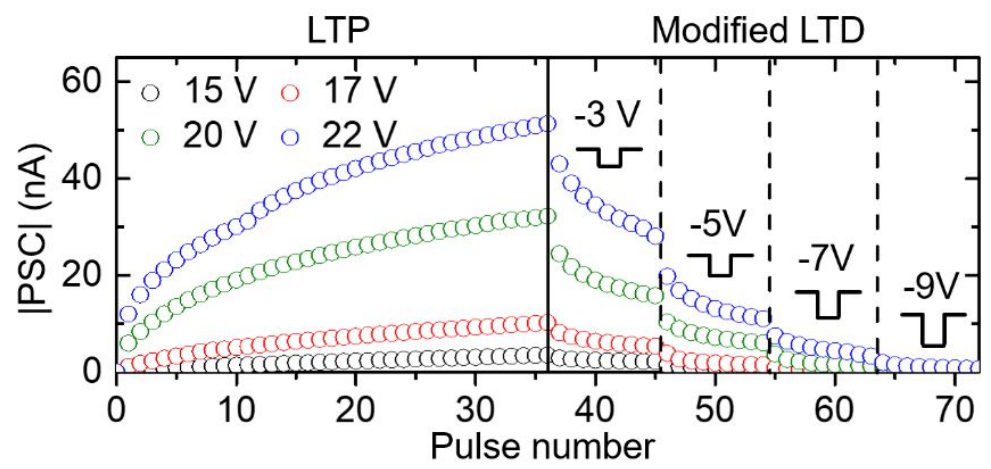

Figure S6. LTP/LTD characteristics of the PTCDI-C $8 / \mathrm{CuPc} / p-6 \mathrm{P}$ synaptic device with four positive $V_{\mathrm{WC}} \mathrm{S}$ $(+15,+17,+20$, and $+22 \mathrm{~V})$ in the LTP region and four negative $V_{\mathrm{WC}} \mathrm{S}(-3,50,-7$, and $-9 \mathrm{~V})$ in the LTD region.
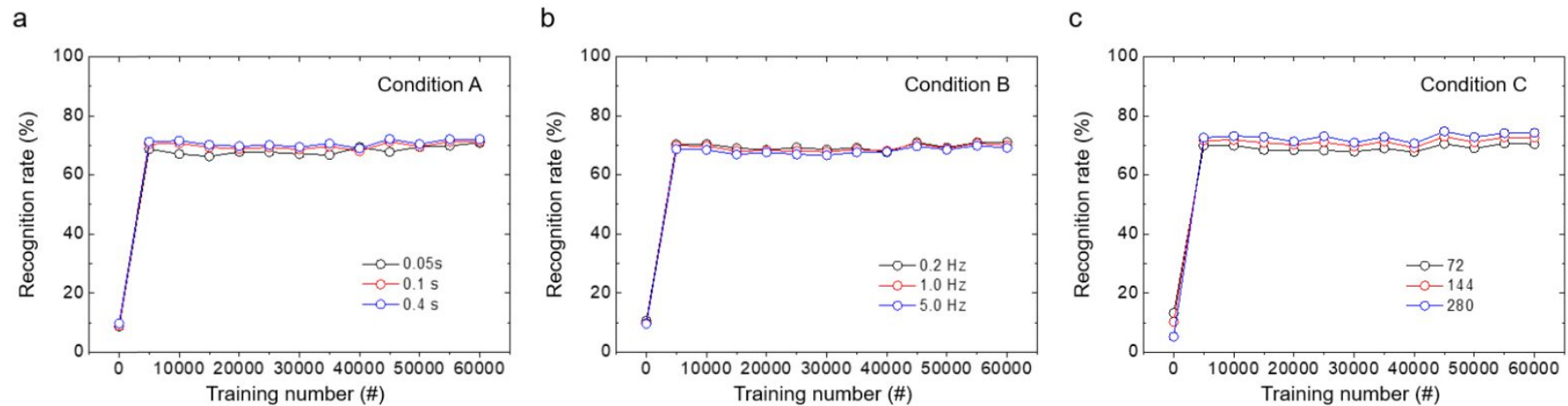

Figure S7. Recognition rate of ANNs trained using various pulse conditions: (a) pulse width, (b) pulse frequency, and (c) pulse number for MNIST digit pattern.

a

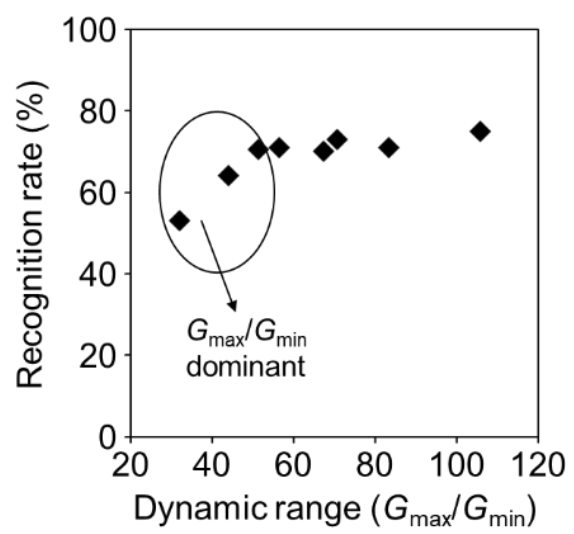

b

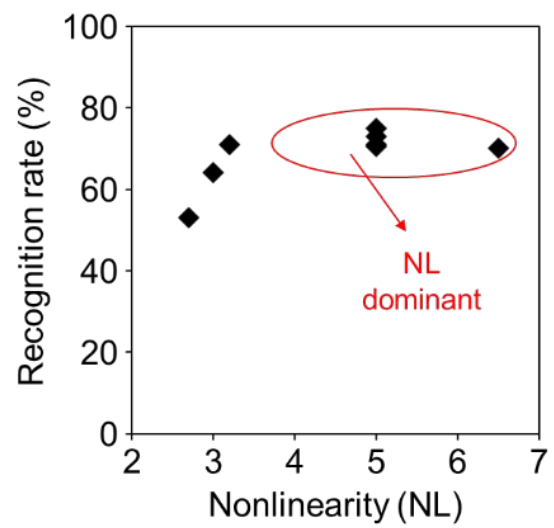

Figure S8. Extracted recognition rates of ANNs according to (a) dynamic range of the maximum/minimum conductance ratio $\left(G_{\max } / G_{\min }\right)$ and (b) nonlinearity (NL) 
Table S1. Extracted Fitting Parameters for Pattern Recognition Tasks.

\begin{tabular}{|c|c|c|c|c|c|c|c|}
\hline & & $\alpha_{P}$ & $\alpha_{D}$ & $\boldsymbol{\beta}_{\mathbf{P}}$ & $\boldsymbol{\beta}_{\mathbf{D}}$ & $\mathbf{G}_{\max }$ & $\mathbf{G}_{\min }$ \\
\hline \multicolumn{2}{|c|}{ Case \#1 } & $1.8 \mathrm{e}-10$ & $9 \mathrm{e}+7$ & 0.1 & 45 & $3.49 \mathrm{e}-9$ & $3.09 \mathrm{e}-11$ \\
\hline \multicolumn{2}{|c|}{ Case \#2 } & $1.75 \mathrm{e}-10$ & $1.5 \mathrm{e}-9$ & 0.1 & 5 & $3.71 \mathrm{e}-9$ & $4.55 \mathrm{e}-11$ \\
\hline \multicolumn{2}{|c|}{ Case \#3 } & $1.73 \mathrm{e}-10$ & $7.5 e-10$ & 0.1 & 3.5 & $3.51 \mathrm{e}-9$ & $4.27 \mathrm{e}-11$ \\
\hline \multirow{3}{*}{$\begin{array}{l}\text { Pulse width } \\
\text { (s) }\end{array}$} & 0.05 & $5.25 \mathrm{e}-11$ & $1.75 \mathrm{e}-10$ & 0.01 & 2.7 & $1.89 \mathrm{e}-9$ & $3.07 \mathrm{e}-11$ \\
\hline & 0.1 & $1.73 \mathrm{e}-10$ & $7.5 e-10$ & 1 & 3 & $3.51 \mathrm{e}-9$ & $4.69 \mathrm{e}-11$ \\
\hline & 0.4 & $5.5 \mathrm{e}-10$ & $1.4 \mathrm{e}-9$ & 1.3 & 3.2 & $8.95 \mathrm{e}-9$ & $3.46 \mathrm{e}-11$ \\
\hline \multirow{3}{*}{$\begin{array}{c}\text { Pulse frequency } \\
(\mathrm{Hz})\end{array}$} & 0.2 & $2 \mathrm{e}-10$ & $3.25 \mathrm{e}-10$ & 1.3 & 2.5 & $3.39 \mathrm{e}-9$ & $4.69 \mathrm{e}-11$ \\
\hline & 1 & $3.2 \mathrm{e}-10$ & $5.5 e-9$ & 1.3 & 5 & $5.49 \mathrm{e}-9$ & $8.39 \mathrm{e}-11$ \\
\hline & 5 & $4.5 \mathrm{e}-10$ & $2.4 \mathrm{e}-8$ & 1.3 & 6.5 & $7.17 \mathrm{e}-9$ & $6.11 \mathrm{e}-11$ \\
\hline \multirow{3}{*}{ Pulse number } & 72 & $3.2 \mathrm{e}-10$ & $5.5 \mathrm{e}-9$ & 1.3 & 5 & $5.47 \mathrm{e}-9$ & $8.39 \mathrm{e}-11$ \\
\hline & 144 & $1.95 \mathrm{e}-10$ & $3.5 \mathrm{e}-9$ & 1 & 5 & $8.32 \mathrm{e}-9$ & $7.9 \mathrm{e}-11$ \\
\hline & 280 & $1.4 \mathrm{e}-10$ & $2.75 e-9$ & 1 & 5 & $1.17 \mathrm{e}-8$ & $7.3 \mathrm{e}-11$ \\
\hline
\end{tabular}


Recently, various studies have been reported to improve the recognition rate of ANNs. Jia et al. increased $G_{\max } / G_{\min }$ significantly from 10.9 to 31.9 without degradation of linearity by using a mixed spike (light and voltage pulses), thereby enhancing the recognition rate for MNIST digit patterns from $49 \%$ to $62 \%$. ${ }^{[\mathrm{S} 1}$ ] Similarly, Sungho et al. improved $G_{\max } / G_{\min }$ from 7.1 to 58.5 by adjusting the amplitude and width of gate voltage pulses and consequently enhanced the recognition rate from $62 \%$ to $70 \%{ }^{[\mathrm{S} 2]}$ Qian reduced the effective NL from 7.49 to 1.38 by employing a unidirectional update method, which uses only the conductance states of LTP region for training ANNs. Owing to this improvement in linearity, the recognition rate increased from $53 \%$ to $70 \% .^{[\mathrm{S} 3]}$

Table S2. Recent studies to improve the recognition rate for MNIST digit images (SLP only).

\begin{tabular}{|c|c|c|}
\hline & Improved characteristics & Recognition rate enhancement \\
\hline $\begin{array}{c}\text { Pulse modulation } \\
\text { (this work) }\end{array}$ & $* \mathrm{NL}_{\mathrm{D}} \downarrow$ & $53 \% \rightarrow 70 \%(17 \% \uparrow)$ \\
\hline Jia et al. ${ }^{[\mathrm{S} 1]}$ & $G_{\max } / G_{\min \uparrow} \uparrow$ & $49 \% \rightarrow 62 \%(13 \% \uparrow)$ \\
\hline Sungho et al. ${ }^{[\mathrm{S} 2]}$ & $G_{\max } / G_{\min } \uparrow$ & $62 \% \rightarrow 70 \%(8 \% \uparrow)$ \\
\hline Qian et al. ${ }^{[\mathrm{S} 3]}$ & Effective $\mathrm{NL} \uparrow$ & $53 \% \rightarrow 70 \%(17 \% \uparrow)$ \\
\hline
\end{tabular}

$* \mathrm{NL}_{\mathrm{D}}$ denotes the NL value of LTD region.

[S1] Sun, J.; Oh, S.; Choi, Y.; Seo, S.; Oh, M. J.; Lee, M.; Lee, W. B.; Yoo, P. J.; Cho, J. H.; Park, J.-H. Optoelectronic Synapse based on IGZO-Alkylated Graphene Oxide Hybrid Structure. Advanced Functional Materials 2018, 28, 1804397.

[S2] Kim, S.; Choi, B.; Lim, M.; Yoon, J.; Lee, J.; Kim, H.-D.; Choi, S.-J. Pattern Recognition Using Carbon Nanotube Synaptic Transistors with an Adjustable Weight Update Protocol. ACS Nano 2017, 11, 3, 2814-2822.

[S3] Qian, C.; Oh, S.; Choi, Y.; Kim, J.-H.; Sun, J.; Huang, H.; Yang, J.; Gao, Y.; Park, J.-H.; Ch o, J. H. Solar-Stimulated Optoelectronic Synapse Based on Organic Heterojunction with Linearly Pot entiated Synaptic Weight for Neuromorphic Computing. Nano Energy 2019, 66, 104095. 